\title{
ON SUBCANONICAL GORENSTEIN VARIETIES AND APOLARITY
}

\author{
PIETRO DE POI AND FRANCESCO ZUCCONI
}

\begin{abstract}
Let $X$ be a codimension 1 subvariety of dimension $>1$ of a variety of minimal degree $Y$. If $X$ is subcanonical with Gorenstein canonical singularities admitting a crepant resolution, then $X$ is Arithmetically Gorenstein and we characterise such subvarieties $X$ of $Y$ via apolarity as those whose apolar hypersurfaces are Fermat.
\end{abstract}

\section{INTRODUCTION}

The Kodaira vanishing theorem does not extend to a normal Gorenstein variety, see [GR70, Section 3.3]. On the other hand, it is known that schemes with Gorenstein canonical singularities have a single sheaf, denoted by $\omega_{X}$, that fits perfectly for both duality and vanishing theorems, see [Kol95, Corollary 11.13].

Then - even if in the context of Mori theory the Gorenstein assumption is too restrictive - to understand normal varieties with Gorenstein (strictly) canonical singularities is quite important and in this paper we prove some theorems on them.

We recall that a variety is called s-subcanonical if the dualising sheaf $\omega_{X}$ exists and $\mathcal{L}^{\otimes s}$ is isomorphic to $\omega_{X}$ where $s \in \mathbb{Z}, \mathcal{L}=\phi^{*} \mathcal{O}_{\mathbb{P}^{N}}(1)$, and $\phi: X \rightarrow \mathbb{P}^{N}$ is an embedding. The ring $\mathcal{R}:=\bigoplus_{i=0}^{\infty} H^{0}\left(X, \mathcal{L}^{\otimes i}\right)$ is known as the canonical ring in the case $s=1$ and as the anticanonical ring in the case $s=-1$. A huge amount of study has been devoted to these rings also in a more general setting. Here it is sufficient to recall [AS78] and Gre82 only.

In this paper we extensively use the concept of arithmetically Cohen-Macaulay projective variety (aCM for short), see Definition 1, and the concept of arithmetically Gorenstein variety (aG for short), see Definition 2 ,

We extend the results of Gre82 with the use of the General Kodaira Vanishing, see Kol95, Theorem 2.17].

We consider normal projective $n$-dimensional aCM varieties $X \subset \mathbb{P}^{N}$ with canonical Gorenstein singularities that are regular (i.e. $h^{1,0}(X)=0$ ) for which $\omega_{X}$ is base point free, the image of the canonical map $\varphi_{\omega_{X}}$ has maximal dimension and $h^{0}\left(X, \omega_{X}\right) \geq n+2$. We show that for such varieties the canonical $\operatorname{ring} \mathcal{R}$ of $X$ is generated in degree $n$ unless the image of the canonical map is a variety of minimal degree, in which case $\mathcal{R}$ is generated by elements of degree at most $n+1$. The above results are also generalised to $s$-subcanonical varieties; see Proposition 1 .

We also give conditions under which a projective variety $X \subset \mathbb{P}^{N}$ with canonical Gorenstein singularities is aG. We show (See Theorem $[$ and Theorem 8 ) that this happens if $X$ is $s$-subcanonical and $\ell$-normal, for all $\ell$ with $0 \leq \ell \leq n+s-1$ and, if $s \geq 0$, satisfies the additional condition that $h^{i}\left(X, \mathcal{O}_{X}(k)\right)=0$ for $1 \leq i \leq n-1$ and $0 \leq k \leq s$.

We point out that this result can be related also to Vak06, Proposition 6.9].

Date: 7th April 2019.

2000 Mathematics Subject Classification. 14J40, 14J45, 13H10, 14N05, $14 \mathrm{~J} 17$.

Key words and phrases. Gorenstein variety, Subcanonical varieties, apolarity. 
In this paper we write that a variety $X$ is $s$-aG if it is aG and $s$-subcanonical, and we call $s$-subcanonically regular a variety which is $s$-aG with Gorenstein canonical singularities. By Theorem 8 we can extend the approach of our previous works DZ11 and DZ2 to this class of varieties. We recall that F. Macaulay proved that an Artinian graded Gorenstein ring and so of socle dimension 1 and degree - say- $k$ can be realised as $A=\frac{\mathbb{C}\left[\partial_{0}, \ldots, \partial_{m}\right]}{F^{\perp}}$, where $F \in \mathbb{C}\left[x_{0}, \ldots, x_{m}\right]$ is a homogeneous polynomial of degree $k$ and $F^{\perp}:=\left\{D \in \mathbb{C}\left[\partial_{0}, \ldots, \partial_{m}\right] \mid D(F)=0\right\}$, where $\mathbb{C}\left[\partial_{0}, \ldots, \partial_{m}\right]$ is the polynomial ring generated by the natural derivations over $\mathbb{C}\left[x_{0}, \ldots, x_{m}\right]$, see Mac16 and [K99, Section 2.3]. We call $F$ the Macaulay polynomial of $A$. Now let $X \subset \mathbb{P}^{N}$ be an $s$-subcanonically regular variety of dimension $n$, with homogeneous coordinate ring $S_{X}$. A choice of $n+1$ independent linear forms $\eta_{0}, \ldots, \eta_{n}$, determines an Artinian graded Gorenstein ring $\frac{S_{X}}{\left(\eta_{0}, \ldots, \eta_{n}\right)}$ of socle of degree $s+n+1$. The Macaulay polynomial of this ring is a form $F_{\eta_{0}, \ldots, \eta_{n}}$ of degree $s+n+1$ in $N-n$ variables. This defines a rational map:

$$
\alpha_{X}: \mathbb{G}(m, N) \rightarrow H_{m, s+n+1}
$$

from the Grassmannian of $m$-planes in $\mathbb{P}^{N}$ (where $m=N-(n+1)$ ) to the space of homogeneous polynomials of degree $s+n+1$ in $\check{\mathbb{P}}^{N}$ modulo the action of $\mathbb{P G L}(m+$ $1, \mathbb{C})$, sending the $m$-plane $\left(\eta_{0}=\ldots=\eta_{n}=0\right)$ to the orbit of $F_{\eta_{0}, \ldots, \eta_{n}}$.

In this paper we prove natural generalisations of the celebrated Noether and Enriques-Petri-Babbage Theorems [ACGH85, §III.3] in the wider context of $s$ subcanonically regular varieties:

Main Theorem. Let $(X, \mathcal{L})$ be a polarised $(k-1)$-dimensional variety, such that $X \subset|\mathcal{L}|^{\vee}=: \check{\mathbb{P}}^{N}$ is an s-subcanonical variety with crepant resolution and with $k>2, k+s>2$; then $X$ is contained as a codimension one subvariety in a rational normal scroll, or a quadric, or a cone on the Veronese surface $v_{2}\left(\mathbb{P}^{2}\right)$ if and only if it is $s$-subcanonically regular and for every $k$-tuple of general sections $\eta_{1}, \ldots, \eta_{k} \in H^{0}(X, \mathcal{L}), F_{\eta_{1}, \ldots, \eta_{k}} \in \mathbb{C}\left[x_{0}, \ldots, x_{N-k}\right]$ is a Fermat hypersurface of degree $(s+k)$.

See Theorem 23. Main Theorem is the first step to study the geometry of an $s$ subcanonically regular variety of dimension $n$ via the behaviour of the rational map $\alpha_{X}: \mathbb{G}(m, N) \rightarrow H_{m, s+n+1}$. For a non-trivial example concerning the canonical curve case see BCN11. Moreover, we stress the fact that, contrary to the curve case - that is $k=2$-it follows, from Main Theorem, that given a variety $X$ of dimension $\geq 2$ with Gorenstein strictly canonical singularities, to be contained as a divisor in a rational normal scroll and to be subcanonical forces $X$ to be aCM (and hence $\mathrm{aG}$ ).

We think that the assumption that the resolution is crepant establishes an interesting link between the theory of singularities and the theory of apolarity. Finally we think that some of the geometry we have described could shed some light on some aspects of Artinian Gorenstein Rings, see CRV01.

\section{Preliminaries}

In this paper we will work with projective varieties and schemes over the complex field $\mathbb{C}$. For us, a variety $X$ will always be irreducible but not necessarily smooth.

2.1. Generalisation of some results of Green. Nowadays the results of Gre82] are easily generalisable to many classes of varieties. For this work, we assume that $X$ is normal with Gorenstein canonical singularities. We will follow closely the exposition of Gre82 indicating the changes to be made to adapt it to our case. 
Let $\phi: X \rightarrow \mathbb{P}^{N}$ be a morphism and set $\mathcal{L}=\phi^{*} \mathcal{O}_{\mathbb{P}^{N}}(1)$. Since $\mathcal{L}$ is base point free, following Gre82, §2], we can form the exact sequence:

$$
0 \rightarrow Q_{0}^{*} \rightarrow H^{0}(X, \mathcal{L}) \otimes \mathcal{O}_{X} \rightarrow \mathcal{L} \rightarrow 0
$$

naturally given by the evaluation map $H^{0}(X, \mathcal{L}) \otimes \mathcal{O}_{X} \rightarrow \mathcal{L}$. Then we see that the natural multiplication map $\mu_{d}: H^{0}(X, \mathcal{L}) \otimes H^{0}(X,(d-1) \mathcal{L}) \rightarrow H^{0}(X, d \mathcal{L})$ fits in the cohomology of the obvious sequence obtained from the sequence (2):

$$
\begin{array}{r}
0 \rightarrow H^{0}\left(X, Q_{0}^{*} \otimes(d-1) \mathcal{L}\right) \rightarrow H^{0}(X, \mathcal{L}) \otimes H^{0}(X,(d-1) \mathcal{L}) \stackrel{\mu_{d}}{\rightarrow} H^{0}(X, d \mathcal{L}) \rightarrow \\
\rightarrow H^{1}\left(X, Q_{0}^{*} \otimes(d-1) \mathcal{L}\right) \rightarrow H^{0}(X, \mathcal{L}) \otimes H^{1}(X,(d-1) \mathcal{L}) \rightarrow \cdots
\end{array}
$$

Letting $C_{d}$ be the coker of $\mu_{d}$ we obtain that:

$$
C_{d} \cong \operatorname{ker}\left(H^{1}\left(X, Q_{0}^{*} \otimes(d-1) \mathcal{L}\right) \rightarrow H^{0}(X, \mathcal{L}) \otimes H^{1}(X,(d-1) \mathcal{L})\right) .
$$

We are ready to prove:

Proposition 1. Let $X$ be an $n$-dimensional variety with normal Gorenstein canonical singularities. Let $\mathcal{L}$ be a line bundle on $X$ such that:

(1) $\mathcal{L}^{\otimes s} \cong \omega_{X}$

(2) $|\mathcal{L}|$ is base point free;

(3) the map associated to $|\mathcal{L}|, \varphi_{|\mathcal{L}|}: X \rightarrow \mathbb{P}^{N}$ is such that $\operatorname{dim}\left(\varphi_{|\mathcal{L}|}(X)\right)=n$;

(4) $\varphi_{|\mathcal{L}|}(X)$ is not a variety of minimal degree and has codimension at least two.

Then the ring $\mathcal{R}:=\oplus_{d=0}^{+\infty} H^{0}(X, d \mathcal{L})$ is generated by elements of degree at most $n+s-1$ if $h^{1}\left(X, \mathcal{O}_{X}\right)=0$.

Proof. Since $X$ is Cohen-Macaulay, see Kol95, Corollary 11.13], we can apply Serre Duality, and then

$$
C_{d}^{*} \cong H^{n-1}\left(X, Q_{0} \otimes(s-d+1) \mathcal{L}\right) / \operatorname{Im}\left(H^{0}(X, \mathcal{L})^{*} \otimes H^{n-1}(X,(s-d+1) \mathcal{L})\right),
$$

The subtle vanishing theorem [Gre82, Theorem 2.14] holds under our hypothesis too. In fact Gre82, Theorem 2.8] holds for a variety with normal Gorenstein canonical singularities since the general Kodaira vanishing is applicable. Now Gre82, Theorem 1.3] is obviously independent of any assumption on the singularities of $X$, while the crucial condition in the proof of [Gre82, Theorem 2.14] is that $X$ is regular. Finally to apply our generalisation of [Gre82, Theorem 2.14], using the same notations as in Gre82, Theorem 2.14], we have to put $p=1, k=n-1$, $n=p+k$; so if $s-d+1=-k$, that is $d=s+n$, then the claim follows verbatim as in the proof of Gre82, Theorem $3.9(3)]$ since $h^{n-1}\left(X, Q_{0} \otimes(1-n) \mathcal{L}\right)=0$ and therefore $C_{s+n}=0$.

Remark 1. Notice that under the assumptions of Proposition 1 we have $C_{d}=0$ if $d \geq s+n$ as in the proof of Gre82, Theorem 3.9 (3)] since we can apply [Gre82, Theorem 2.8] (2) to obtain $h^{n-1}\left(X, Q_{0} \otimes(-n) \mathcal{L}\right)=0$ and [Gre82, Theorem 2.8] (1) to obtain $h^{n-1}\left(X, Q_{0} \otimes(-m) \mathcal{L}\right)=0$ where $m>n$.

2.2. Arithmetically Gorenstein schemes. Let us fix a closed subscheme $Z$ of $\mathbb{P}^{N}$ of dimension $n \geq 1$ and a system $x_{0}, \ldots, x_{N}$ of projective coordinates. Let $\mathcal{I}_{Z}$ be the sheaf of ideals of $Z$. The module $M^{r}(Z):=\oplus_{t \in \mathbb{Z}} H^{r}\left(\mathbb{P}^{N}, \mathcal{I}_{Z}(t)\right), 1 \leq r \leq n$, is called the $r$-th Hartshorne-Rao module of $Z$.

Definition 1. Let $Z \subset \mathbb{P}^{N}$ be a a closed subscheme of dimension $n$. Then $Z$ is said to be arithmetically Cohen-Macaulay ( $a C M$ for short) if $N-n$ is equal to the length of a minimal free resolution of its homogeneous coordinate ring

$$
S_{Z}:=\frac{S}{I_{Z}}
$$


as an $S$-module, where $S:=\mathbb{C}\left[x_{0}, \ldots, x_{N}\right]$ and $I_{Z}:=\oplus_{t \in \mathbb{Z}} H^{0}\left(\mathbb{P}^{N}, \mathcal{I}_{Z}(t)\right)$ is the homogeneous ideal of $Z$.

A zero-dimensional scheme is automatically aCM, see Mig98, page 10].

Proposition 2. A scheme $Z \subset \mathbb{P}^{N}$ of dimension $\geq 1$ is aCM if and only if $M^{r}(Z)=0$ for all $1 \leq r \leq n$.

Proof. See for example [Mig98, 1.2.2 and 1.2.3].

Definition 2. Let $Z \subset \mathbb{P}^{N}$ be a closed subscheme. $Z$ is said to be arithmetically Gorenstein ( $a G$ for short) if it is aCM and the last free module of a minimal free resolution of $S_{Z}$ has rank 1.

Definition 3. A subscheme $Z \subset \mathbb{P}^{N}$ is said to be subcanonical if there exists an integer $s \in \mathbb{Z}$ such that $\omega_{Z} \cong \mathcal{O}_{Z}(s)$, where $\omega_{Z}$ is the dualising sheaf of $Z$ (which exists by [Har83, Proposition III.7.5]).

Note that an aCM scheme is aG if and only if it is subcanonical. The dualising sheaf of an $n$-dimensional projective scheme $Z \subset \mathbb{P}^{N}$ is

$$
\omega_{Z}=\mathcal{E}_{x} t_{\mathcal{O}_{\mathbb{P}} N}^{N-n}\left(\mathcal{O}_{Z}, \omega_{\mathbb{P}^{N}}\right)
$$

which is the sheafification of the canonical model $\operatorname{Ext}_{S}^{N-n}\left(S_{Z}, S\right)(-N-1)$. By Serre's correspondence, if $Z$ is aCM then the canonical model of the last free module of the minimal free resolution of $S_{Z}$ has rank 1 exactly when $\omega_{Z} \cong \mathcal{O}_{Z}(s)$ for some $s \in \mathbb{Z}$. More precisely we have the following:

Proposition 3. If $Z$ is an aCM closed subscheme of $\mathbb{P}^{N}$, then the following are equivalent:

(1) $Z$ is $a G$;

(2) $\omega_{Z} \cong \mathcal{O}_{Z}(s)$ for some integer $s$;

(3) the minimal free resolution of $S_{Z}$ is self-dual, up to a twist.

Proof. See [Mig98, Proposition 4.1.1].

We need the following:

Definition 4. Let $Z \subset \mathbb{P}^{N}$ be a projective closed subscheme. We say that $Z$ is $j$-normal, with $j \in \mathbb{Z}, j \geq 0$, if the natural restriction map

$$
H^{0}\left(\mathbb{P}^{N}, \mathcal{O}_{\mathbb{P}^{N}}(j)\right) \rightarrow H^{0}\left(Z, \mathcal{O}_{Z}(j)\right)
$$

is surjective. We say that $Z$ is projectively normal ( $P N$ for short) if it is $j$-normal $\forall j \in \mathbb{Z}, j \geq 0$.

The following is an easy characterisation of aCM schemes among $P N$ ones:

Proposition 4. Let $X \subset \mathbb{P}^{N}$ be an n-dimensional $P N$ scheme. Then $X$ is aCM if and only if

$$
h^{i}\left(X, \mathcal{O}_{X}(j)\right)=0, \quad \text { for } 0<i<n \text { and } \forall j \in \mathbb{Z} .
$$

Proof. By Proposition 2, $X$ is an aCM-scheme iff $h^{r}\left(\mathbb{P}^{N}, \mathcal{I}_{X}(j)\right)=0$ for every $j \in \mathbb{Z}$ and $1 \leq r \leq n$. We consider the cohomology of the standard sequence for $X \subset \mathbb{P}^{N}$ :

$$
0 \rightarrow \mathcal{I}_{X}(j) \rightarrow \mathcal{O}_{\mathbb{P}^{N}}(j) \rightarrow \mathcal{O}_{X}(j) \rightarrow 0 .
$$

Since $X$ is PN, then by Bott's theorem it follows $h^{1}\left(\mathbb{P}^{N}, \mathcal{I}_{X}(j)\right)=0$ where $j \in \mathbb{Z}$. Using Bott's theorem again it follows that $h^{i+1}\left(\mathbb{P}^{N}, \mathcal{I}_{X}(j)\right)=h^{i}\left(X, \mathcal{O}_{X}(j)\right)$ where $0<i<n$ and $j \in \mathbb{Z}$.

Classically a variety $X$ is called irregular if $h^{1}\left(X, \mathcal{O}_{X}\right)>0$. 
Corollary 5. let $X \subset \mathbb{P}^{N}$ be an $n$-dimensional irregular variety. If $n \geq 2, X$ cannot be $a G$.

In the case of subcanonical varieties we can get more than what is in Proposition 4. thanks to the general Kodaira vanishing theorem and some generalisations of the results in Gre82 proved above, see Proposition 1.

Theorem 6. Let $X \subset \mathbb{P}^{N}$ be a canonical $n$-dimensional $\ell$-normal variety where $1 \leq \ell \leq n$. Assume that $X$ has normal Gorenstein canonical singularities and that $h^{i}\left(X, \mathcal{O}_{X}\right)=0$ for all $1 \leq i \leq n-1$. Then $X$ is $a G$.

Proof. Since $X$ is canonical then $\mathcal{O}_{X}(1) \cong \omega_{X}$. Hence by Proposition 3 we only need to show that $X$ is aCM.

First of all, without loss of generality we can suppose that $X$ has codimension at least two.

The cohomology of $0 \rightarrow \mathcal{I}_{X}(k) \rightarrow \mathcal{O}_{\mathbb{P}^{N}}(k) \rightarrow \mathcal{O}_{X}(k) \rightarrow 0$ gives:

$$
0 \rightarrow H^{0}\left(\mathbb{P}^{N}, \mathcal{I}_{X}(k)\right) \rightarrow H^{0}\left(\mathbb{P}^{N}, \mathcal{O}_{\mathbb{P}^{N}}(k)\right) \rightarrow H^{0}\left(X, \mathcal{O}_{X}(k)\right) \rightarrow H^{1}\left(\mathbb{P}^{N}, \mathcal{I}_{X}(k)\right) \rightarrow 0
$$

and $H^{i}\left(X, \mathcal{O}_{X}(k)\right) \cong H^{i+1}\left(\mathbb{P}^{N}, \mathcal{I}_{X}(k)\right)$ for $0<i<n$ and for every $k \in \mathbb{Z}$. By the general Kodaira vanishing theorem, $h^{i}\left(X, \mathcal{O}_{X}(k)\right)=0$ for $k<0, i<n$. Moreover, Serre duality holds and it gives:

$$
h^{i}\left(X, \mathcal{O}_{X}(k)\right)=h^{n-i}\left(X, \mathcal{O}_{X}(1-k)\right)
$$

and therefore, since $h^{i}\left(X, \mathcal{O}_{X}\right)=0$, we deduce $h^{i}\left(X, \mathcal{O}_{X}(k)\right)=0$ for $1 \leq i \leq n-1$, $\forall k \in \mathbb{Z}$.

It remains to show that $X$ is $\mathrm{PN}$. By our $\ell$-normality hypothesis, it remains to prove that $h^{1}\left(\mathbb{P}^{N}, \mathcal{I}_{X}(k)\right)=0$, if $k>n$. Since $h^{1}\left(X, \mathcal{O}_{X}\right)=0$, we can apply Proposition 1 with $s=1$. Then the canonical ring of $X$ is generated in degree $n$. Now we show that $X$ is $(n+1)$-normal, the case where $k \geq n+2$ works by induction on $k$ in the same vein. Since $\omega_{X}=\mathcal{O}_{X}(1)$ and since we have assumed that $X$ is $n$-normal we can put the natural homomorphism $\operatorname{Sym}^{n+1} H^{0}\left(X, \omega_{X}\right) \rightarrow H^{0}\left(X, \omega_{X}^{\otimes n+1}\right)$ and the multiplication map $H^{0}\left(X, \omega_{X}\right) \otimes H^{0}\left(X, \omega_{X}^{\otimes n}\right) \rightarrow H^{0}\left(X, \omega_{X}^{\otimes n+1}\right)$ in the following exact commutative diagram:

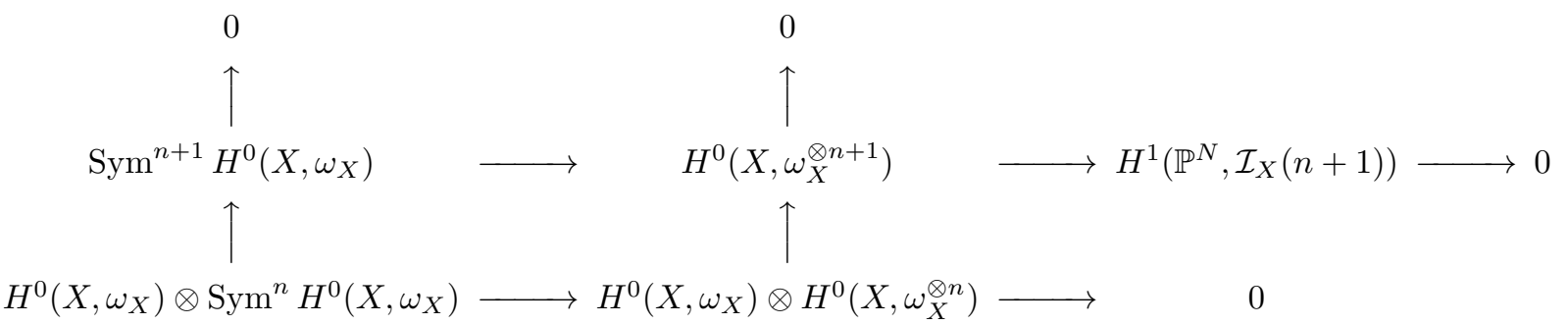

obtained by (3) .

Let $\xi \in H^{1}\left(\mathbb{P}^{N}, \mathcal{I}_{X}(n+1)\right)$. By surjectivity, there exists $\gamma \in H^{0}\left(X, \omega_{X}^{\otimes n+1}\right)$ such that $\gamma \mapsto \xi$. From the surjectivity in the second column, there exists an $\alpha \in H^{0}\left(X, \omega_{X}\right) \otimes \operatorname{Sym}^{n} H^{0}\left(X, \omega_{X}\right)$ such that $\alpha \mapsto \gamma$. Therefore, by the surjectivity in the second row, there exists an $\bar{\alpha} \in H^{0}\left(X, \omega_{X}\right) \otimes \operatorname{Sym}^{n} H^{0}\left(X, \omega_{X}\right)$ such that $\bar{\alpha} \mapsto \alpha$. Let $\bar{\gamma} \in \operatorname{Sym}^{n+1} H^{0}\left(X, \omega_{X}\right)$ be the image of $\bar{\alpha}$. By commutativity, $\bar{\gamma} \mapsto \gamma$. Then by the exactness of the first row, $\xi$ must be zero.

Because of the history of the topic, we like to recall the following corollary of Theorem 6.

Corollary 7. Let $X \subset \mathbb{P}^{N}$ be a smooth canonical regular 2-normal surface. Then $X$ is $a C M$ and therefore is $a G$. 
The result of Theorem 6 is also true in the $s$-subcanonical case with a mild extra hypothesis; but in this case the assumption on singularities is interesting.

Theorem 8. Let $s \in \mathbb{Z}$. Let $X \subset \mathbb{P}^{N}$ be a s-subcanonical n-dimensional $\ell$-normal, for all $\ell$ such that $1 \leq \ell \leq n+s-1$, variety with normal Gorenstein canonical singularities. If $s \geq 0$ assume also that $h^{i}\left(X, \mathcal{O}_{X}(k)\right)=0$, for all $i, k \in \mathbb{Z}$ such that $1 \leq i \leq n-1$ and $0 \leq k \leq s$. Then $X$ is $a G$.

Proof. The proof is identical to the proof of Theorem [6 except that in this case $\omega_{X}=\mathcal{O}_{X}(s)$ and this causes some trivial shifts in the indices.

We give the following:

Definition 5. Let $s \in \mathbb{Z}$. An $s$-aG variety $X \subset \mathbb{P}^{N}$ with normal Gorenstein canonical singularities will be called an $s$-subcanonically regular variety.

Thanks to Theorem 8 , being an $s$-subcanonically regular variety is equivalent to requiring that for all $1 \leq i \leq \operatorname{dim}(X)-1$ and for all $0 \leq k \leq s, h^{i}\left(X, \mathcal{O}_{X}(k)\right)=0$ and $h^{1}\left(\mathbb{P}^{N}, \mathcal{I}_{X}(\ell)\right)=0$, for all $1 \leq \ell \leq \operatorname{dim}(X)+s-1$.

\section{On SubCANONicAlly REgular VARIETIES}

Theorem 8 makes it possible to extend the results of [DZ11] and [DZ2] to some $s$-subcanonically regular varieties; see also the introduction.

To do this, we recall a few facts about apolarity theory.

3.1. Apolarity. An excellent reference for apolarity is the notes by A. Geramita, Ger96]. Let $S:=\mathbb{C}\left[x_{0}, \ldots, x_{N}\right]$ be the polynomial ring in $(N+1)$-variables. The algebra of partial derivatives on $S$,

$$
T:=\mathbb{C}\left[\partial_{0}, \ldots, \partial_{N}\right], \quad \partial_{i}:=\frac{\partial}{\partial_{x_{i}}},
$$

acts on the monomials by:

$$
\partial^{a} \cdot x^{b}= \begin{cases}a !\left(\begin{array}{l}
b \\
a
\end{array}\right) x^{b-a} & \text { if } b \geq a \\
0 & \text { otherwise }\end{cases}
$$

where $a, b$ are multiindices, $\left(\begin{array}{l}b \\ a\end{array}\right)=\prod_{i}\left(\begin{array}{c}b_{i} \\ a_{i}\end{array}\right), a !:=\prod_{i} a_{i}$ ! etc.

We can think of $S$ as the algebra of partial derivatives on $T$ by defining

$$
x^{a} \cdot \partial^{b}= \begin{cases}a !\left(\begin{array}{l}
b \\
a
\end{array}\right) \partial^{b-a} & \text { if } b \geq a \\
0 & \text { otherwise. }\end{cases}
$$

These actions define a perfect pairing between the forms of degree $d$ in $S$ and $T$, $\forall d \in \mathbb{N}:$

$$
S_{d} \times T_{d} \rightarrow \mathbb{C}
$$

Indeed, this is nothing but the extension of the duality between vector spaces: if $V:=S_{1}$, then $T_{1}=V^{*}$.

This perfect paring shows the natural duality between $\mathbb{P}^{N}:=\operatorname{Proj}(S)$ and $\check{\mathbb{P}}^{N}=$ $\operatorname{Proj}(T)$. More precisely, if $\left(c_{0}, \ldots, c_{N}\right) \in \check{\mathbb{P}}^{N}$, this gives $f_{c}:=\sum_{i} c_{i} x_{i} \in S_{1}$, and if $D \in T_{a}$,

$$
D \cdot f_{c}^{b}= \begin{cases}a !\left(\begin{array}{l}
b \\
a
\end{array}\right) D(c) f_{c}^{b-a} & \text { if } b \geq a \\
0 & \text { otherwise }\end{cases}
$$

in particular, if $b \geq a$

$$
0=D \cdot f_{c}^{b} \Longleftrightarrow D(c)=0
$$

where $D(c)$ is the value of the polynomial $D$ at $c:=\left(c_{0}, \ldots, c_{N}\right)$. 
Definition 6. We say that two forms, $f \in S$ and $g \in T$ are apolar if

$$
g \cdot f=f \cdot g=0 .
$$

Let $f \in S_{d}$ and $F:=V(f) \subset \mathbb{P}^{N}$ the corresponding hypersurface; let us now define

$$
F^{\perp}:=\{D \in T \mid D \cdot f=0\}
$$

and

$$
A^{F}:=\frac{T}{F^{\perp}} .
$$

Lemma 9. The ring $A^{F}$ is Artinian Gorenstein of socle of degree d.

Proof. See [K99, §2.3 page 67].

Definition 7. $A^{F}$ is called the apolar Artinian Gorenstein ring of $F$.

The Macaulay Lemma asserts that any Artinian Gorenstein ring of socle of degree $d$ is apolar to a hypersurface of degree $d$; more precisely:

Lemma 10. The map

$$
F \mapsto A^{F}
$$

is a bijection between the hypersurfaces $F \subset \mathbb{P}^{N}$ of degree $d$ and graded Artinian Gorenstein quotient rings

with socle of degree $d$.

$$
A:=\frac{T}{I}
$$

Proof. See [K99, Lemma 2.12 page 67].

If $A=A^{F}$ then the polynomial $F$ is called the Macaulay polynomial of $A$.

3.1.1. Varieties of sum of powers. Consider a hypersurface $F=V(f) \subset \mathbb{P}^{N}$ of degree $d$.

Definition 8. A subscheme $\Gamma \subset \check{\mathbb{P}}^{N}$ is said to be apolar to $F$ if

$$
I(\Gamma) \subset F^{\perp} \text {. }
$$

The Apolarity Lemma holds:

Lemma 11. Let us consider the linear forms $\ell_{1}, \ldots, \ell_{s} \in S_{1}$ and let us denote by $L_{1}, \ldots, L_{s} \in \check{\mathbb{P}}^{N}$ the corresponding points in the dual space and by $\Gamma:=\left\{L_{1}, \ldots, L_{s}\right\} \subset$ $\check{\mathbb{P}}^{N}$ the corresponding zero-dimensional subscheme. Then

$\Gamma$ is apolar to $F=V(f), \Longleftrightarrow \exists \lambda_{1}, \ldots, \lambda_{s} \in \mathbb{C}^{*}$ such that $f=\lambda_{1} \ell_{1}^{d}+\ldots+\lambda_{s} \ell_{s}^{d}$ If $s$ is minimal, then it is called the Waring number of $F$.

Proof. See [IK99, Lemma 1.15 page 12].

3.2. Macaulay polynomials of $s$-subcanonically regular varieties. In DZ11, we studied the special case of the canonical curve $C \subset \check{\mathbb{P}}^{g-1}$ of the map introduced in (1). In fact it is a well-known result that $C$ is aG (see ACGH85, page 117]). Therefore, if we take two general linear forms $\eta_{1}, \eta_{2} \in\left(\mathcal{R}_{C}\right)_{1}=H^{0}\left(\omega_{C}\right)$, then $T:=\frac{\mathcal{R}_{C}}{\left\langle\eta_{1}, \eta_{2}\right\rangle}$ is Artinian Gorenstein, and its values of the Hilbert function are $1, g-2, g-2,1$. In particular, the socle degree of $T$ is 3 , and by the Macaulay Lemma, this defines a hypercubic in $\operatorname{Proj}\left(T^{*}\right)$. In this way we obtain the rational $\operatorname{map} \alpha_{C}: \mathbb{G}(g-3, g-1) \rightarrow H_{g-3,3}$.

Theorem 12. Let $X \subset \mathbb{P}^{N}$ be an s-subcanonically regular variety of dimension $n$. Let $\eta_{0}, \ldots, \eta_{n}$ be $n+1$ general linear forms on $\mathbb{P}^{N}$. Then the graded $\mathbb{C}$-algebra $A:=\frac{S_{X}}{\left\langle\eta_{0}, \ldots, \eta_{n}\right\rangle}$ is of degree $s+n+1$. 
Proof. By Theorem 8 and by Proposition 3 , the homogeneous coordinate ring $S_{X}$ is aG, i.e. it is the homogeneous coordinate ring of an aG scheme.

Therefore, also $A=S_{X} /\left(\eta_{0}, \ldots, \eta_{n}\right)$ is aG since $\eta_{0}, \ldots, \eta_{n}$ is a regular sequence: see for example BH93, Proposition 3.1.19(b)]. The ring $A$ is obviously graded so by Proposition 3(3) it has symmetric Hilbert function since it is aG. By symmetry, the socle of $A$ is of dimension 1. Now, it remains to prove that the socle of $A$ is of degree $s+n+1$. Let $K_{A}$ be the canonical model of $A$, see BH93. Definition 3.6.8 page 139, also page 140] and let $a(A)$ be the $a$-invariant of $A$, see [BH93, Definition 3.6.13]. By Proposition 3(2), $K_{X}=S_{X}(s)$, then $K_{A}=A(s+n+1)$ since BH93, Corollary 3.6.14]. In particular, $a(A)=s+n+1$ by BH93, Corollary 3.6.14]. This means $A_{s+n+1} \neq 0$ and $A_{i}=0$ for $i \geq s+n+2$ (see the remark which follows BH93, Theorem 3.6.19]).

Remark 2. With simple but tedious calculations we could find the values of the Hilbert function of $A$ of the preceding theorem.

By Theorem 12 it easily follows that an $s$-subcanonically regular variety $X \subset \mathbb{P}^{N}$ of dimension $n$, defines the map $\alpha_{X}: \mathbb{G}(m, N) \rightarrow H_{m, s+n+1}$ presented in the introduction, see (1).

It is natural to extract pieces of information on the geometry of $X$ by the nature of this map and, vice versa, to understand some features of some Artinian Gorenstein graded $\mathbb{C}$-algebras. We are informed that the realm of Artinian Gorenstein graded $\mathbb{C}$-algebras is huge, but the case where $X$ is a curve is quite important, see CRV01.

In this paper we concentrate mostly on the geometrical aspects of the problem. The first step, in the light of DZ11, is to understand which are the varieties whose Macaulay polynomials are Fermat hypersurfaces.

3.3. Example: the case of hypersurfaces. We analyse now a way to obtain subcanonical varieties via hypersurfaces of the $M$-dimensional projective space and Veronese embeddings of $\mathbb{P}^{M}$.

So, let us consider a hypersurface $Y \subset \mathbb{P}^{M}$ of degree $s n+M+1>0, s \in \mathbb{Z}$, $n, M \in \mathbb{N}$; therefore, $Y \in|(s n+M+1) H|$, where $H$ is the hyperplane divisor on $\mathbb{P}^{M}$. Assume that $Y$ has only normal Gorenstein canonical singularities. By adjunction we have:

$$
\omega_{Y}=\mathcal{O}_{\mathbb{P}^{M}}(\operatorname{snH}) \otimes_{\mathcal{O}_{\mathbb{P}} M} \mathcal{O}_{Y}
$$

Now, consider the $n$-th embedding of $\mathbb{P}^{M}, v_{n}: \mathbb{P}^{M} \rightarrow \mathbb{P}^{N}$, where $N:=\left(\begin{array}{c}n+M \\ n\end{array}\right)-1$. $V_{n}:=v_{n}\left(\mathbb{P}^{M}\right)$ is a Veronese variety, and it is well-known that it is an aCM variety of degree $n^{M}$.

Since $V_{n}$ is aCM, it follows that $X:=v_{n}(Y) \subset V_{n}$ is aCM also:

Proposition 13. Let $Y \subset \mathbb{P}^{M}$ be a hypersurface of degree sn $+M+1$ with normal Gorenstein canonical singularities. Let $X$ be its $n$-tuple Veronese embedding $X:=$ $v_{n}(Y) \subset V_{n} \subset \mathbb{P}^{N}$, with $N=\left(\begin{array}{c}n+M \\ n\end{array}\right)-1$. Then $X$ is a $G$ and s-subcanonically regular.

Proof. We need to show that $h^{i}\left(\mathbb{P}^{N}, \mathcal{I}_{X}(j)\right)=0$ for all $j \in \mathbb{Z}, 1 \leq i \leq M-1$. 
By the above inclusions we can construct the following commutative and exact diagram of sheaves:

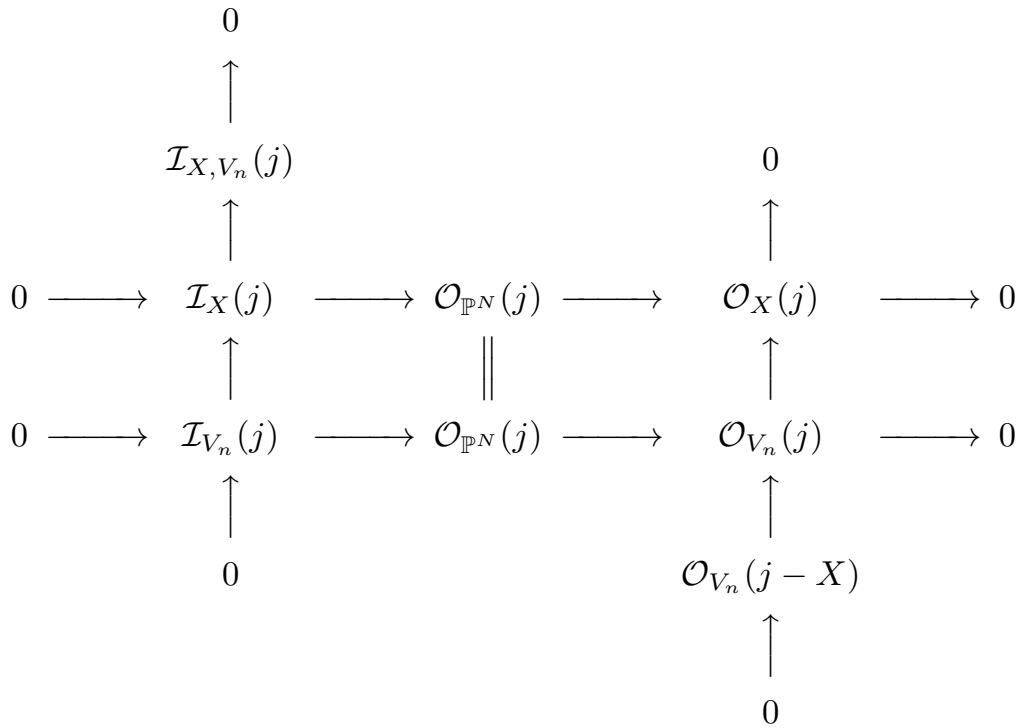

By the above diagram $\mathcal{I}_{X, V_{n}}(j) \cong \mathcal{O}_{V_{n}}(j-X)$, and then $h^{i}\left(\mathbb{P}^{N}, \mathcal{I}_{V_{n}}(j)\right)=0$ for all $j \in \mathbb{Z}, 1 \leq i \leq M$ because $V_{n}$ is aCM. Therefore it is sufficient to show that $h^{i}\left(\mathbb{P}^{N}, \mathcal{O}_{V_{n}}(j-X)\right)=0$ for all $j \in \mathbb{Z}, 1 \leq i \leq M-1$.

Since $v_{n}: \mathbb{P}^{M} \rightarrow V_{n} \subset \mathbb{P}^{N}$ is an embedding, we are reduced to show only that $h^{i}\left(\mathbb{P}^{M}, \mathcal{O}_{\mathbb{P}^{M}}(j n-s n-M-1)\right)=0$ for all $j \in \mathbb{Z}, 1 \leq i \leq M-1$, which is well-known (see Har83, III.5.1]).

Moreover, by (4), $X$ is also aG and $s$-subcanonical.

Corollary 14. Let $C \subset \mathbb{P}^{K}$ be a cone over the $n$-tuple Veronese embedding $V_{n} \subset$ $\mathbb{P}^{N}$ of $\mathbb{P}^{M}$, with $N=\left(\begin{array}{c}n+M \\ n\end{array}\right)-1$. Let $Z \subset C$ be a codimension one subvariety with normal Gorenstein canonical singularities which is s-subcanonical. Then $Z$ is $s-a G$ and it is linearly equivalent to a cone over the image of a hypersurface $Y \subset \mathbb{P}^{M}$ of degree $s n+M+1$ under the $n$-tuple Veronese embedding.

Proof. By Har83, Exercise II.6.3], $\operatorname{Pic}(C) \cong \operatorname{Pic}\left(V_{n}\right)$, and the isomorphism is given by the projection from the vertex of the cone. Now, $\operatorname{Pic}\left(V_{n}\right) \cong \operatorname{Pic}\left(\mathbb{P}^{M}\right)=\mathbb{Z}$, so $Z$ is linearly equivalent to a cone over the image of a hypersurface $Y \subset \mathbb{P}^{M}$ of degree $s n+M+1$ under the $n$-tuple Veronese embedding. By Proposition [13, this cone $D$ is aCM (recall Definition 1 and the fact that a cone and its base have isomorphic homogeneous coordinate rings) and hence $s$-aG. Moreover, since $X$ is linearly equivalent to $D$, it follows that $h^{i}\left(\mathbb{P}^{N}, \mathcal{O}_{C}(j-X)\right)=h^{i}\left(\mathbb{P}^{N}, \mathcal{O}_{C}(j-D)\right)$ for all $j \in \mathbb{Z}$, hence the assertion.

Moreover, we deduce immediately:

Proposition 15. Let $Y \subset \mathbb{P}^{M}$ be a hypersurface of degree sn $+M+1$ with normal Gorenstein canonical singularities. Let $A:=\frac{S_{X}}{\left\langle\eta_{0}, \ldots, \eta_{M-1}\right\rangle}$ be the Artinian graded Gorenstein ring corresponding to the s-subcanonical variety $X:=v_{n}(Y) \subset \mathbb{P}^{N}$, with $\eta_{0}, \ldots, \eta_{M-1}$ general linear forms on $\mathbb{P}^{N}$. Then the Macaulay polynomial of $A$ is an $(s+M)$-tic of Waring number at most $n^{M}$ (i.e. it is the sum of at most $n^{M}(s+M)$-th powers of linear forms).

Proof. The estimate for the Waring number is given by the degree of the Veronese variety $V_{n}=v_{n}\left(\mathbb{P}^{M}\right) \supset X$. 
3.4. Complete intersections. Perhaps the easiest way to obtain aG varieties is doing complete intersections in projective space. In fact, if a variety $X \subset \mathbb{P}^{N}$ is the complete intersection of $c$ hypersurfaces, $F_{1} \ldots, F_{c}$ of degrees $d_{1}, \ldots, d_{c}$, then, by adjunction, we have that $\left.\omega_{X} \cong \mathcal{O}_{\mathbb{P}^{N}}\left(-N-1+\sum_{i=1}^{c} d_{i}\right)\right|_{X}$. We set $E:=$ $\oplus_{i=1}^{c} \mathcal{O}_{\mathbb{P}^{N}}\left(-d_{i}\right)$. We have the Koszul complex:

$$
0 \rightarrow \wedge^{c} E \rightarrow \cdots \rightarrow E \rightarrow \mathcal{I}_{X} \rightarrow 0,
$$

and noticing that the intermediate cohomology of $\wedge^{i} E$ is zero, we deduce that $X$ is aCM also. Therefore, $X$ is $s$-aG, with $s:=-N-1+\sum_{i=1}^{c} d_{i}$.

Lemma 16. Let $X$ be a codimension one subcanonical subvariety of a quadric hypersurface $Q \subset \mathbb{P}^{N}$. Then $X$ is $a G$.

Proof. Let $r$ be the rank of the quadric $Q$; if $r \geq 4$, then, by Klein's theorem, see Har83, Exercise II.6.5(d)], $X$ is a complete intersection, and therefore it is aG.

If $r=2$, then $\operatorname{Pic}(Q) \cong \operatorname{Pic}(C)=\mathbb{Z} P$, where $C$ is a smooth conic and $P$ is the class of a (closed) point of $C$, and the first isomorphism is given by the projection of the cone onto its base; moreover, the class of the hyperplane section $H$ of $Q$ is double the generator, i.e. $H \equiv 2 P$ (where $\equiv$ is the linear equivalence of divisors) see Har83, Exercises II.6.3 and II.6.5(c)]. In particular, we can think of $Q$ as a cone with base $C \subset \mathbb{P}^{2}$. $X$ is linearly equivalent to the subscheme $D$ given by $d$ rulings of the cone, where $d:=\operatorname{deg}(X)$, i.e. the span of the vertex of $Q$ with the $d$ points $X \cap \mathbb{P}^{2}$. By adjunction, $X \cap \mathbb{P}^{2} \subset C$ is subcanonical, and hence $\mathrm{aG}$, since it is zero-dimensional. Therefore, $D$ is aG. Now, since $X \equiv D$, then $h^{i}\left(\mathbb{P}^{N}, \mathcal{O}_{Q}(j-X)\right)=h^{i}\left(\mathbb{P}^{N}, \mathcal{O}_{Q}(j-D)\right)$ for all $j \in \mathbb{Z}$, hence the assertion.

If $r=3$, then $\operatorname{Pic}(Q) \cong\left(Q^{\prime}\right) \cong \mathbb{Z} F_{1} \oplus \mathbb{Z} F_{2}$, where $Q^{\prime} \subset \mathbb{P}^{3}$ is a smooth quadric and $F_{1}, F_{2}$ are the classes of the two rulings of $Q^{\prime}$, and the first isomorphism is given by the projection of the cone onto its base; moreover, the class of the hyperplane section $H$ of $Q$ is $H \equiv F_{1}+F_{2}$ (see again Har83, Exercises II.6.3 and II.6.5(c)]). In particular, we can think of $Q$ as a cone with base $Q^{\prime} \subset \mathbb{P}^{3} . X$ is linearly equivalent to the cone over a curve $C \subset Q^{\prime}$; moreover, we can identify $C$ with $X \cap Q^{\prime} \subset \mathbb{P}^{3}$, and hence $C$ is subcanonical. Suppose that $C$ is $s$-subcanonical and $[C]=a F_{1}+b F_{2}$. Then $a=b=s+2$, because on $Q$ every effective divisor moves, so $C$ is linearly equivalent to a complete intersection, which is aCM, and we can conclude as above that $X$ is aCM and therefore aG.

3.5. 1-codimensional varieties in rational normal scrolls. For fixing some notations, we recall some basic facts about rational normal scrolls.

3.5.1. Rational normal scrolls. By definition, a rational normal scroll (RNS for short) of type $\left(a_{1}, \ldots, a_{k}\right)$, is the image $S_{a_{1}, \ldots, a_{k}}$ of the $\mathbb{P}^{k-1}$-bundle $\pi: \mathbb{P}(\mathcal{E})=$ $\mathbb{P}\left(\mathcal{O}_{\mathbb{P}^{1}}\left(a_{1}\right) \oplus \cdots \oplus \mathcal{O}_{\mathbb{P}^{1}}\left(a_{k}\right)\right) \rightarrow \mathbb{P}^{1}$, under the morphism $j: \mathbb{P}(\mathcal{E}) \rightarrow \mathbb{P}^{N}$ defined by the tautological bundle $\mathcal{O}_{\mathbb{P}(\mathcal{E})}(1)$. We can arrange the integers $0 \leq a_{1} \leq a_{2} \leq \ldots \leq a_{k}$ and notice that $N=k-1+f$ where we set $f:=\sum_{i=1}^{k} a_{i}=\operatorname{deg}\left(S_{a_{1}, \ldots, a_{k}}\right)$. If $a_{1}=a_{2}=\ldots=a_{\ell}=0$ and $a_{\ell+1} \neq 0$ where $1 \leq \ell<k$ then $S_{a_{1}, \ldots, a_{k}}$ is a cone of vertex $V$ of dimension $\ell-1$. Notice, in particular, that if $a_{1}=a_{2}=\ldots=a_{k-1}=0$ and $a_{k}=1$ then $S_{a_{1}, \ldots, a_{k}}=\mathbb{P}^{N}$ where $N=k$. Since our theory is for varieties $X$ contained in a projective space, in the case of varieties $X \subset S_{a_{1}, \ldots, a_{k}}$ we need to study the morphism $j: Y \rightarrow X$ induced by $j: \mathbb{P}(\mathcal{E}) \rightarrow S_{a_{1}, \ldots, a_{k}} \subset \mathbb{P}^{N}$ on the $j$-strict transform $Y$ of $X$. We follow the well-written exposition on Weil divisors on rational normal scrolls Fer01.

It is well known and easy to see that $j: \mathbb{P}(\mathcal{E}) \rightarrow S_{a_{1}, \ldots, a_{k}} \subset \mathbb{P}^{N}$ is a rational resolution of the singularity $V$. In particular the Weil divisors are Cartier divisors on $\mathbb{P}(\mathcal{E})$ but this is not true in general for $S_{a_{1}, \ldots, a_{k}}$. Write $\mathrm{Cl}(X)$ and $\mathrm{CaCl}(X)$ for the divisor 
class group and the group of Cartier divisors modulo principal divisors of $X$, as in Har83. Since $\operatorname{Codim}\left(V, S_{a_{1}, \ldots, a_{k}}\right) \geq 2$, restriction to the complement of $V$ gives an isomorphism $\mathrm{Cl}\left(S_{a_{1}, \ldots, a_{k}}\right) \rightarrow \mathrm{Cl}\left(S_{a_{1}, \ldots, a_{k}} \backslash V\right)$ by Har83, Proposition II.6.5]. Since $j$ is an isomorphism over $j^{-1}\left(S_{a_{1}, \ldots, a_{k}} \backslash V\right)$, we have a surjective homomorphism $J: \operatorname{CaCl}(\mathbb{P}(\mathcal{E})) \rightarrow \mathrm{Cl}\left(S_{a_{1}, \ldots, a_{k}}\right)$ which is an isomorphism if $\operatorname{Codim}\left(V, S_{a_{1}, \ldots, a_{k}}\right)>2$ and has kernel isomorphic to $\mathbb{Z}$, spanned by the class of the exceptional divisor $E$ of $j$, if $\operatorname{Codim}\left(V, S_{a_{1}, \ldots, a_{k}}\right)=2$.

In Fer01 $J(D)$ is called the strict image of a Cartier divisor $D \subset \mathbb{P}(\mathcal{E})$. In particular if $[H]$ and $[F]$ are respectively the class of the tautological divisor and the class of the fibre of $\pi$, then $\operatorname{Pic}(\mathbb{P}(\mathcal{E}))=[H] \mathbb{Z} \oplus[F] \mathbb{Z}$. We denote by $\sim$ the numerical equivalence of divisors. It easily follows that:

Corollary 17. (1) If $\operatorname{Codim}\left(V, S_{a_{1}, \ldots, a_{k}}\right)>2$, then $\operatorname{Cl}\left(S_{a_{1}, \ldots, a_{k}}\right)=J[H] \mathbb{Z} \oplus$ $J[F] \mathbb{Z}$

(2) if $\operatorname{Codim}\left(V, S_{a_{1}, \ldots, a_{k}}\right)=2$, then $E \sim H-f F$ and $\mathrm{Cl}\left(S_{a_{1}, \ldots, a_{k}}\right)=J[F] \mathbb{Z}$.

We remark that the Weil divisor $J(F)$ on $S_{a_{1}, \ldots, a_{k}}$ is not Cartier if $V \neq \emptyset$ since, as in the standard case of the quadric cone, $J(F)$ is not locally principal in a neighbourhood of $V$. Clearly the theory of Weil divisors on $S_{a_{1}, \ldots, a_{k}}$ splits in two cases according to the codimension of $V$. For what we need, we only stress that given a closed irreducible reduced subscheme $X \subset S_{a_{1}, \ldots, a_{k}}$ of pure codimension 1 with no embedded components, the proper transform $Y \subset \mathbb{P}(\mathcal{E})$ is the closure $\overline{j^{-1}\left(X \cap\left(\left(S_{a_{1}, \ldots, a_{k}} \backslash V\right)\right)\right.}$. Hence, by linearity, the proper transform $Y$ is defined for every Weil divisor $X$ on $S_{a_{1}, \ldots, a_{k}}$, and $J(Y)=X$. Moreover if $\operatorname{Codim}\left(V, S_{a_{1}, \ldots, a_{k}}\right)>2$, then there exists a unique Cartier divisor $Y$ such that $J(Y)=X$, but if $\operatorname{Codim}\left(V, S_{a_{1}, \ldots, a_{k}}\right)=2$ then $J(Y+m E)=X$ for every $m \in \mathbb{Z}$.

Finally to treat the group $\operatorname{Div}\left(S_{a_{1}, \ldots, a_{k}}\right)$ of divisorial sheaves on a singular scroll in terms of $\operatorname{Pic}(\mathbb{P}(\mathcal{E}))$ we recall that $\operatorname{Div}\left(S_{a_{1}, \ldots, a_{k}}\right)=\frac{\mathrm{Cl}\left(S_{a_{1}, \ldots, a_{k}}\right)}{\equiv}$ where $\equiv$ is the linear equivalence and, if $\operatorname{Codim}\left(V, S_{a_{1}, \ldots, a_{k}}\right)>2$, then $j_{\star}: \operatorname{Pic}(\mathbb{P}(\mathcal{E})) \rightarrow \operatorname{Div}\left(S_{a_{1}, \ldots, a_{k}}\right)$ is an isomorphism. To study also the case $\operatorname{Codim}\left(V, S_{a_{1}, \ldots, a_{k}}\right)=2$ we recall that letting $\mathcal{F}^{\vee \vee}$ be the double dual of a sheaf on a normal scheme, the homomorphism $J$ induces a homomorphism, denoted by the same letter, $J: \operatorname{Pic}(\mathbb{P}(\mathcal{E})) \rightarrow \operatorname{Div}\left(S_{a_{1}, \ldots, a_{k}}\right)$ given by $\mathcal{O}_{\mathbb{P}(\mathcal{E})}(Y) \mapsto j_{*}\left(\mathcal{O}_{\mathbb{P}(\mathcal{E})}(Y)\right)^{\vee \vee}$. The homomorphism $J$ is an isomorphism if $\operatorname{Codim}\left(V, S_{a_{1}, \ldots, a_{k}}\right)>2$. Hence with a minor effort we see that the theory of Cartier divisors and numerical equivalence on $\operatorname{Pic}(\mathbb{P}(\mathcal{E}))$ goes parallel to the theory of Weil divisors and linear equivalence on $\operatorname{Div}\left(S_{a_{1}, \ldots, a_{k}}\right)$. In particular

Lemma 18. Let $j: \mathbb{P}(\mathcal{E}) \rightarrow S_{a_{1}, \ldots, a_{k}} \subset \mathbb{P}^{N}$ be the desingularisation of a singular scroll of vertex $V$ such that $\operatorname{Codim}\left(V, S_{a_{1}, \ldots, a_{k}}\right)>2$. Then it holds:

(1) let $X \equiv a J(H)+b J(F)$ be a Weil divisor on $S_{a_{1}, \ldots, a_{k}}$; then $Y \sim a H+b F$ for the unique Cartier divisor on $\mathbb{P}(\mathcal{E})$ such that $J(Y)=X$;

(2) $j_{*} \mathcal{O}_{\mathbb{P}(\mathcal{E})}(Y)=\mathcal{O}_{S_{a_{1}, \ldots, a_{k}}}(X)$;

(3) $\mathcal{O}_{S_{a_{1}, \ldots, a_{k}}}(X)=\left(\mathcal{O}_{S_{a_{1}, \ldots, a_{k}}}(X)\right)^{\vee \vee}$.

Proof. See [Fer01, Note 3.14 and Corollary 3.20].

For the special case where $\operatorname{Codim}\left(V, S_{a_{1}, \ldots, a_{k}}\right)=2$ there is the problem that given a Weil divisor $X$ it is not necessarily true that $j_{*} \mathcal{O}_{\mathbb{P}(\mathcal{E})}(Y+m E)=\mathcal{O}_{S_{a_{1}, \ldots, a_{k}}}(X)$ for any $m \in \mathbb{Z}$. To choose a good element in the set $J^{-1}[X]=\{[Y+m E] \mid m \in \mathbb{Z}\}$, in [Fer01, the rational total transform of $X$ is defined as the rational divisor $Y+q E$ where $q$ is uniquely obtained by the relation $(Y+q E) \cdot E \cdot H^{k-2}=0$. Then assuming first that $X$ is effective and that $Y \sim a H+b F$, it is easy to see that $q=\frac{b}{f}$ (recall $\left.f=\sum a_{i}\right)$ so we can define a unique element $X^{*}=Y+n_{q} E \in J^{-1}(X)$ called the integral total transform of $X$ where $n_{q}$ is the smallest integer $\geq q$. 
Lemma 19. Let $j: \mathbb{P}(\mathcal{E}) \rightarrow S_{a_{1}, \ldots, a_{k}} \subset \mathbb{P}^{N}$ be the desingularisation of a singular scroll of vertex $V$ such that $\operatorname{Codim}\left(V, S_{a_{1}, \ldots, a_{k}}\right)=2$. Then the integral total transform $X^{*}$ of a Weil divisor $X$ on $S_{a_{1}, \ldots, a_{k}}$ is uniquely determined by the linear equivalence class. Moreover:

(1) let $X \equiv d J(F)$ where $d \geq 0$; then $X^{*} \sim(m+1) H+(f-h-1) F$ where $d-1=m f+h\left(m \geq-1\right.$ and $0 \leq h<f, m+1=a+n_{q}$ and $f-h-1=$ $\left.f n_{q}-b\right)$

(2) let $X \equiv d J(F)$ where $d<0$; then $\mathcal{O}_{S_{a_{1}, \ldots, a_{k}}}(X) \simeq \mathcal{O}_{S_{a_{1}, \ldots, a_{k}}}(-(m+$ 1) $J(H)+(f-h-1) J(F)) \simeq \mathcal{O}_{S_{a_{1}, \ldots, a_{k}}}(-m J(H)-(h+1) J(F))$;

(3) let $X \equiv d J(F)$ where $d \in \mathbb{Z}$; then $j_{*} \mathcal{O}\left(X^{*}\right)=\mathcal{O}_{S_{a_{1}, \ldots, a_{k}}}(X)$.

Recall that $S_{a_{1}, \ldots, a_{k}}$ is aCM, and that in Sch86] it is proved that the dualising sheaf $\omega_{S_{a_{1}, \ldots, a_{k}}}$ satisfies:

$$
\omega_{S_{a_{1}, \ldots, a_{k}}}=j_{*} \mathcal{O}_{\mathbb{P}(\mathcal{E})}\left(K_{\mathbb{P}(\mathcal{E})}\right)
$$

where the canonical class of $\mathbb{P}(\mathcal{E})$ is given by:

$$
K_{\mathbb{P}(\mathcal{E})}=-k H+(N-k-1) F .
$$

3.5.2. Crepant s-subcanonical varieties in a RNS. We consider now 1-codimensional subvarieties contained in rational normal scrolls and we ask under which conditions they are $s$-subcanonical. To obtain the classification we assume that if $X \subset S_{a_{1}, \ldots, a_{k}}$ is a Gorenstein irreducible $s$-subcanonical variety then the morphism $j: \mathbb{P}(\mathcal{E}) \rightarrow$ $S_{a_{1}, \ldots, a_{k}} \subset \mathbb{P}^{N}$ induces a crepant morphism $j_{\mid Y}: Y \rightarrow X$ on the strict transform $Y$ of $X$. For the notion of crepant morphism see Rei83. The crepant condition is a natural one in the context of $s$-subcanonically regular varieties.

Lemma 20. Let $s \in \mathbb{Z}$ and let $X \subset S_{a_{1}, \ldots, a_{k}} \subset \mathbb{P}^{N}, k>2$, be a Gorenstein irreducible s-subcanonical variety. Let $Y$ be the $j$-proper transform of $X$. Assume that the morphism $j_{\mid Y}: Y \rightarrow X$ is crepant, that is $j^{*}\left(\omega_{X}\right)=\omega_{Y}$, and that $Y \sim$ $a H+b F$. If $k>-s$ then $s+k=a$ and $b=k+1-N$ or $a=k+s+1, b=0$ and $N=k$ (i.e. $X$ is a hypersurface of $S_{a_{1}, \ldots, a_{k}}=\mathbb{P}^{N}$ where $a_{1}=\ldots=a_{k-1}=0$ and $\left.a_{k}=1\right)$.

Proof. The assumption that $X$ is $s$-subcanonical means that $\mathcal{O}_{X}(s)=\omega_{X}$. The assumption that $X$ is Gorenstein implies that we can use adjunction theory to write $\omega_{X}=\mathcal{O}_{X}(X) \otimes \mathcal{O}_{X} \omega_{S_{a_{1}, \ldots, a_{k}}}$. Now we distinguish two cases according to the codimension of the vertex $V$ of the RNS $S_{a_{1}, \ldots, a_{k}}$. To ease reading we remind the reader that $H \in H^{0}\left(\mathbb{P}(\mathcal{E}), \mathcal{O}_{\mathbb{P}(\mathcal{E})}(1)\right)$. We denote by $E$ a general effective divisor of the linear system $\left|H-\pi^{*} a_{k} F\right|$.

First case. $\operatorname{Codim}\left(V, S_{a_{1}, \ldots, a_{k}}\right)>2$.

Let us define a divisor $\eta$ on $\mathbb{P}(\mathcal{E})$ as

$$
\eta:=(a-k-s) H+(b+N-k-1) F .
$$

First we show that $\eta$ is an effective divisor which does not move. By definition

$$
\eta-Y=(-k-s) H+(N-k-1) F \sim K_{\mathbb{P}(\mathcal{E})}-s H .
$$

By Lemma 18, numerical equivalence over $\mathbb{P}(\mathcal{E})$ translates to linear equivalence over $S_{a_{1}, \ldots, a_{k}}$ and, again by Lemma 18 we have $j_{*} \mathcal{O}_{Y}(\eta)=\mathcal{O}_{X}$ since $j_{*} \mathcal{O}_{Y}(\eta)=\omega_{X}(-s)$. Consider the following exact sequence:

$$
0 \rightarrow \mathcal{O}_{\mathbb{P}(\mathcal{E})}(\eta-Y) \rightarrow \mathcal{O}_{\mathbb{P}(\mathcal{E})}(\eta) \rightarrow \mathcal{O}_{Y}(\eta) \rightarrow 0 .
$$

Since $s>-k$ then, by (5), $\mathcal{O}_{\mathbb{P}(\mathcal{E})}(\eta-Y)$ has no sections, and we have that $h^{0}\left(\mathbb{P}(\mathcal{E}), \mathcal{O}_{\mathbb{P}(\mathcal{E})}(\eta-Y)\right)=0$. 
Now it is enough to show that $h^{0}\left(\mathbb{P}(\mathcal{E}), \mathcal{O}_{\mathbb{P}(\mathcal{E})}(\eta)\right)=1$. Since $h^{0}\left(Y, \mathcal{O}_{Y}(\eta)\right)=$ $h^{0}\left(X, j_{*} \mathcal{O}_{Y}(\eta)\right)=h^{0}\left(X, \mathcal{O}_{X}\right)=1$, then it is sufficient to show that $h^{1}\left(\mathcal{O}_{\mathbb{P}(\mathcal{E})}(\eta-\right.$ $Y))=0$. Since $N=k-1+f$ and $\operatorname{Pic}(\mathbb{P}(\mathcal{E}))=[H] \mathbb{Z} \oplus[F] \mathbb{Z}$ we can write $\eta-$ $Y=(-k-s) H-f F$. Thus we have to show that $h^{1}\left(\mathbb{P}(\mathcal{E}), \mathcal{O}_{\mathbb{P}(\mathcal{E})}(-k-s) \otimes\right.$ $\mathcal{O}_{\mathbb{P}(\mathcal{E})}((-f F))=0$. By Har83, Exercise III.8.4(a)] we deduce that $R^{i} \pi_{*}\left(\mathcal{O}_{\mathbb{P}(\mathcal{E})}(-k-\right.$ $s))=0 \forall i>0$ (recall that $-k-s<0$ ). Then by Har83, Exercise III.8.1], $H^{1}\left(\mathbb{P}(\mathcal{E}), \mathcal{O}_{\mathbb{P}(\mathcal{E})}(-k-s)\right) \cong H^{1}\left(\mathbb{P}^{1}, \pi_{*}\left(\mathcal{O}_{\mathbb{P}(\mathcal{E})}(-k-s)\right)\right)$, but by again Har83, Exercise III.8.4(a)], $\pi_{*}\left(\mathcal{O}_{\mathbb{P}(\mathcal{E})}(-k-s)\right)=0$. Since $\left.\mathcal{O}_{\mathbb{P}(\mathcal{E})}(F)\right) \cong \pi^{*} \mathcal{O}_{\mathbb{P}^{1}}(1)$, we can conclude by the projection formula ( Har83, Exercise III.8.3]).

It then follows that $\eta$ is an effective divisor such that $h^{0}\left(\mathbb{P}(\mathcal{E}), \mathcal{O}_{\mathbb{P}(\mathcal{E})}(\eta)\right)=1$. By a trivial numerical computation it follows that these conditions imply that there exists an $m \in \mathbb{N}$ such that $\eta \sim m E=m\left(H-a_{k} F\right)$ and $a_{k}>a_{k-1}$. Moreover, by induction starting from the sequence

$$
0 \rightarrow \mathcal{O}_{Y}(\eta-Y) \rightarrow \mathcal{O}_{Y}(\eta) \rightarrow \mathcal{O}_{Y_{\mid Y}}(\eta) \rightarrow 0,
$$

we get $\eta \cdot Y^{k-1}=0$. Then the numerical condition $\eta \cdot Y^{k-1}=0$ is equivalent to

$$
m a^{k-2}\left[a \cdot \sum_{i=1}^{k-1} a_{i}+(k-1) b\right]=0
$$

since $H^{k}=\operatorname{deg} S_{a_{1}, \ldots, a_{k}}=\sum_{i=1}^{k} a_{i}=f$. By Equation (6), it follows that either $m=0$, which is the claim, or

$$
b=\frac{a\left(a_{k}-f\right)}{k-1} .
$$

If we proceed as we did for $Y$, with $E=H-a_{k} F$, again by induction, starting from the sequence

$$
0 \rightarrow \mathcal{O}_{E}(\eta-Y) \rightarrow \mathcal{O}_{E}(\eta) \rightarrow \mathcal{O}_{E_{\mid E}}(\eta) \rightarrow 0,
$$

we deduce $\eta \cdot E^{k-1}=0$. From this equation, we infer

$$
b=a\left((k-1) a_{k}-f\right) .
$$

If $a \neq 0$, we have, from Equations (7) and (8), that

$$
(k-2)\left(a_{k} k-f\right)=0 ;
$$

since $k>2$, we have that $f=k a_{k}$, which cannot happen, since we have supposed that $a_{k}>a_{k-1}$.

Second case. $\operatorname{Codim}\left(V, S_{a_{1}, \ldots, a_{k}}\right)=2$.

We have denoted by $E$ the exceptional divisor of $j: \mathbb{P}(\mathcal{E}) \rightarrow S_{a_{1}, \ldots, a_{k}}$. The argument used above shows that $j_{*} \mathcal{O}_{\mathbb{P}(\mathcal{E})}(\eta)=\mathcal{O}_{S_{a_{1}, \ldots, a_{k}}}$ then, by Corollary 17 (2) and by Lemma 19, we have that $(J[\eta])^{*}=0$. In particular there exists an $m \in \mathbb{Z}$ such that $\eta \sim m E$. Then $-s-k+a=m$ and $b+N-k-1=m f$ and

$$
\eta=m E \sim K_{\mathbb{P}(\mathcal{E})}+Y-s H .
$$

In particular since $\mathcal{O}_{Y}(s H)=j^{*} \mathcal{O}_{X}(s)$ we have $\mathcal{O}_{Y}(m E) \simeq \omega_{Y}-j^{*} \omega_{X}=0$. Hence $b=0$ or $m=0$.

Assume $m \neq 0$. By Equation (10) we have that $-s-k+a=m$ and $N-k-1=$ $-m f$. Since $N=f+k-1$ it follows that $(m+1) f=2$ and the only solution-since $f \geq 0$ and we have assumed $m \neq 0$-is $f=1$ and $m=1$.

Remark 3. The case $k=2$ in Lemma 20 was considered in [DZ2, Lemma 15].

Moreover, if $X$ is smooth, it is well known that $k \geq-s$ and $s=-k$ if and only if our variety is $X$ is a $\mathbb{P}^{k-1}$. The same results holds if $X$ is normal and Gorenstein: see Fuj90, Proposition 3.4, Paragraph 4.13 and Theorem 5.15]. 
We can sum up the above general results in the following:

Proposition 21. Let $k \in \mathbb{N}$ be such that $k>2$, and let $s \in \mathbb{Z}$ be such that $k>-s$. Let $X \subset S_{a_{1}, \ldots, a_{k}}$ be a Gorenstein irreducible 1-codimensional subvariety and let $Y \subset \mathbb{P}(\mathcal{E})$ be its $j$-proper transform. Then $X$ is general in its linear equivalence class and s-subcanonical, and $j_{\mid Y}: Y \rightarrow X$ is crepant, if and only if either $Y$ is a general element of $|(s+k) H+(2-f) F|$, or $k=N$ and $Y$ is a hypersurface of degree $(s+k+1)$.

Proof. By Lemma 20 we have only to show that if $Y \in|(s+k) H+(k+1-N) F|$ or $Y \in|(s+k+1) H|$ and $N=k$ is general then $X=j(Y)$ is an $s$-subcanonical Gorenstein irreducible 1-codimensional subvariety and $j_{\mid Y}: Y \rightarrow X$ is crepant. In the case where $Y \in|(s+k+1) H|$ the morphism $S_{a_{1}, \ldots, a_{k}} \rightarrow \mathbb{P}^{N}$ is the blow-up along the a codimension 2 linear subspace of $\mathbb{P}^{N}$. Hence $Y$ is the total transform of a general hypersurface $X$ of degree $(s+k+1)$. Now the assumption that $Y$ is general forces that $X$ is smooth. If $\operatorname{Codim}\left(V, S_{a_{1}, \ldots, a_{k}}\right)>2$ then the claim follows. If $\operatorname{Codim}\left(V, S_{a_{1}, \ldots, a_{k}}\right)=2$ and $f \geq 2$ then by the argument of the second case of the proof of Lemma 20 we have that, taking $Y \in|(s+k) H+(2-f) F|$, it necessarily follows by adjunction that $j_{Y}: Y \rightarrow X=j(Y)$ is crepant.

3.5.3. On aG-subvarieties in a RNS. Next, we show that an $s$-subcanonical, 1codimensional subvariety $X \subset S_{a_{1}, \ldots, a_{k}}$ such that $j_{\mid Y}: Y \rightarrow X$ is crepant is aG:

Proposition 22. Let $k \in \mathbb{N}$ be such that $k>2$ and let $s \in \mathbb{Z}$ be such that $k>$ -s. Let $X \subset S_{a_{1}, \ldots, a_{k}}$ be a Gorenstein irreducible 1-codimensional subvariety, $s$ subcanonical, general in its linearly equivalence class, and such that $j_{\mid Y}: Y \rightarrow X$ is crepant, where $Y \subset \mathbb{P}(\mathcal{E})$ is its $j$-proper transform. Then $X$ is $a G$.

Proof. Set $S:=S_{a_{1}, \ldots, a_{k}} \subset \mathbb{P}^{N}$. By the natural inclusions $X \subset S \subset \mathbb{P}^{N}$ we can construct the following exact and commutative diagram of sheaves:

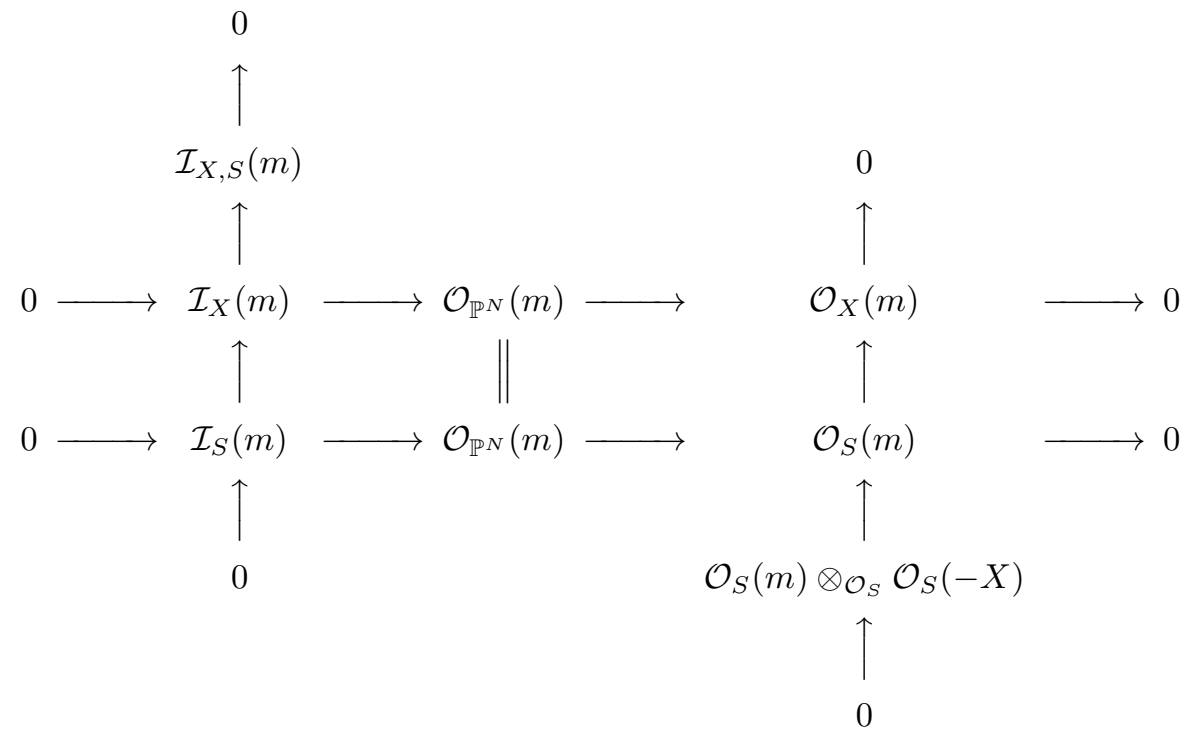

We need to show that $h^{i}\left(\mathbb{P}^{N}, \mathcal{I}_{X}(m)\right)=0$ for all $m \in \mathbb{Z}$ and $1 \leq i \leq k-1$. Set $\mathcal{O}_{S}(m-X):=\mathcal{O}_{S}(m) \otimes \mathcal{O}_{S} \mathcal{O}_{S}(-X)$. By the above diagram, $\mathcal{I}_{X, S}(m) \cong \mathcal{O}_{S}(m-X)$ and, since $S$ is aCM, we have $h^{i}\left(\mathbb{P}^{N}, \mathcal{I}_{S}(m)\right)=0$ for all $m \in \mathbb{Z}, 1 \leq i \leq k$; therefore it is sufficient to show that $h^{i}\left(S, \mathcal{O}_{S}(m-X)\right)=0$ for all $m \in \mathbb{Z}, 1 \leq i \leq k-1$.

First case. $\operatorname{Codim}\left(V, S_{a_{1}, \ldots, a_{k}}\right)>2$ or $N>k$. 
This assumption, with Proposition 21, gives $\mathcal{O}_{S}(m-X)=j_{*} \mathcal{O}_{\mathbb{P}(\mathcal{E})}\left(K_{\mathbb{P}(\mathcal{E})}+(m-\right.$ s) $H$ ). It also gives the crucial inequality: $f=N-k+1 \geq 2$.

Now, since $K_{\mathbb{P}(\mathcal{E})}+(m-s) H \sim(m-s-k) H+(f-2) F$, then by the projection formula (see Har83, exercise III.8.3]) applied to the morphism $j$, we have that $R^{i} j_{*} \mathcal{O}_{\mathbb{P}(\mathcal{E})}\left(K_{\mathbb{P}(\mathcal{E})}+(m-s) H\right)=R^{i} j_{*} \mathcal{O}_{\mathbb{P}(\mathcal{E})}((f-2) F) \otimes_{\mathcal{O}_{S}} \mathcal{O}_{S}(m-s-k)$. The variety $W$ contracted by $j$ is a rational scroll and, since $f \geq 2$, we have both $H^{i}\left(W, \mathcal{O}_{W}((f-\right.$ $2) F)$ ) $=0$ and hence $R^{i} j_{*} \mathcal{O}_{\mathbb{P}(\mathcal{E})}((f-2) F)=0$, for $1 \leq i \leq k-1$. By Har83, Exercise III.8.1], we then have that $H^{i}\left(S, \mathcal{O}_{S}(m-X)\right)=H^{i}\left(\mathbb{P}(\mathcal{E}), \mathcal{O}_{\mathbb{P}(\mathcal{E})}\left(K_{\mathbb{P}(\mathcal{E})}+\right.\right.$ $(m-s) H))$ ), where $m \in \mathbb{Z}$ and $i \geq 0$. To show the claim we need to show that $h^{i}\left(\mathbb{P}(\mathcal{E}), \mathcal{O}_{\mathbb{P}(\mathcal{E})}\left(K_{\mathbb{P}(\mathcal{E})}+\ell H\right)\right)=0$, where $-s \leq \ell \leq 0$. In fact, for all $\ell \in \mathbb{Z}$, $1 \leq i \leq k-1$ it holds that $h^{i}\left(\mathbb{P}(\mathcal{E}), \mathcal{O}_{\mathbb{P}(\mathcal{E})}\left(K_{\mathbb{P}(\mathcal{E})}+\ell H\right)\right)=0$. If $\ell>0$, then $h^{i}\left(\mathbb{P}(\mathcal{E}), \mathcal{O}_{\mathbb{P}(\mathcal{E})}\left(K_{\mathbb{P}(\mathcal{E})}+\ell H\right)\right)=0$, by the Kodaira vanishing theorem. If $\ell \leq 0$, then it is sufficient to prove, by Serre duality, that $h^{i}\left(\mathbb{P}(\mathcal{E}), \mathcal{O}_{\mathbb{P}(\mathcal{E})}(t H)\right)=0$, with $t \geq 0$, $1 \leq i \leq k-1$. Let $\pi$ be, as usual, the natural projection map, $\pi: \mathbb{P}(\mathcal{E}) \rightarrow \mathbb{P}^{1}$; first of all, we have that $R^{i} \pi_{*} \mathcal{O}_{X}(t H)=0$ if $t \geq 0,1 \leq i \leq k-1$ (see for example Har83, Exercise III.8.4(a)]). But then $H^{i}\left(\mathbb{P}(\mathcal{E}), \mathcal{O}_{\mathbb{P}(\mathcal{E})}(t H)\right)=H^{i}\left(\mathbb{P}^{1}, \pi_{*} \mathcal{O}_{\mathbb{P}(\mathcal{E})}(t H)\right)$ (see for example [Har83, Exercise III.8.1]) and we conclude, since $\pi_{*} \mathcal{O}_{\mathbb{P}(\mathcal{E})}(\ell H) \cong$ $\operatorname{Sym}^{t}\left(\mathcal{O}_{\mathbb{P}^{1}}\left(a_{1}\right) \oplus \cdots \oplus \mathcal{O}_{\mathbb{P}^{1}}\left(a_{k}\right)\right)$ (again by Har83, Exercise III.8.4(a)]). We have shown that $X$ is aG.

Second case. $\operatorname{Codim}\left(V, S_{a_{1}, \ldots, a_{k}}\right)=2$ and $f=1$.

In this case $j: S_{0, \ldots, 0,1} \rightarrow \mathbb{P}^{k}$ and by Proposition 21, $Y \in|(s+1+k) H|$. Hence the claim is easy.

With the hypothesis of Proposition 22, we can give a converse to Proposition 21. that is we can characterise $s$-subcanonically regular varieties with a crepant resolution. This is the Main Theorem of the introduction.

Theorem 23. Let $k \in \mathbb{N}$ be such that $k>2$ and let $s \in \mathbb{Z}$ be such that $k+s>$ 2. Let $(X, \mathcal{L})$ be a polarised $(k-1)$-dimensional variety, such that $X \subset|\mathcal{L}|^{\vee}=$ : $\check{\mathbb{P}}^{N}$ is an s-subcanonical variety with crepant resolution; then $X$ is contained as a codimension one subvariety in a rational normal $k$-dimensional scroll $S_{a_{1}, \ldots, a_{k}}$ or a quadric or a cone on the Veronese surface $v_{2}\left(\mathbb{P}^{2}\right)$ if and only if it is $s$-subcanonically regular and for every $k$-tuple of general sections $\eta_{1}, \ldots, \eta_{k} \in H^{0}(X, \mathcal{L}), F_{\eta_{1}, \ldots, \eta_{k}} \in$ $\mathbb{C}\left[x_{0}, \ldots, x_{N-k}\right]$ is a Fermat hypersurface of degree $(s+k)$.

Proof. Since for $N=k$ there is nothing to prove, we can suppose $N>k$.

If $X$ is contained as a divisor in a variety $S$ of minimal degree, then by Lemma 16. by Corollary 14 and by Proposition 22, $X$ is $s$-subcanonically regular. Take $k$ general sections $\eta_{1}, \ldots \eta_{k} \in H^{0}(X, \mathcal{L})$ and consider $\Gamma:=S \cap V\left(\eta_{1}, \ldots, \eta_{k}\right) \subset \check{\mathbb{P}}^{N-k}$. This is a zero-dimensional scheme of length $\operatorname{deg} S$ and, because $S$ is aCM, the (saturated) homogeneous ideal $I(\Gamma)$ of $\Gamma$ is obtained by adding the linear forms $\eta_{1}, \ldots, \eta_{k}$ to $I(S)$, so $I(\Gamma)=\left(I(S), \eta_{1}, \ldots, \eta_{k}\right)$.

Then by the Apolarity Lemma 11, $\Gamma$ is apolar to a Fermat hypersurface $F_{\eta_{1}, \ldots, \eta_{k}} \in$ $\mathbb{C}\left[x_{0}, \ldots, x_{N-k}\right]$ (of degree $s+k$, by Theorem [12), since $S$ is of minimal degree $(N-k+1)$ which is also the number of independent linear forms of $\mathbb{C}\left[x_{0}, \ldots, x_{N-k}\right]$.

Conversely, let us suppose that our $s$-subcanonical variety $X$ is such that for every $k$-tuple of general sections $\eta_{1}, \ldots, \eta_{k} \in H^{0}(X, \mathcal{L})$, there is a zero-dimensional scheme of length $\operatorname{deg} S, \Gamma_{\eta_{1}, \ldots, \eta_{k}} \subset \mathbb{P}^{N-k}:=V\left(\eta_{1}, \ldots, \eta_{k}\right)$ with $I\left(\Gamma_{\eta_{1}, \ldots, \eta_{k}}\right) \subset$ $I\left(X, \eta_{1}, \ldots, \eta_{k}\right)$, or, in other words, by the Apolarity Lemma 11] $\Gamma_{\eta_{1}, \ldots, \eta_{k}}$ is apolar to a Fermat $(s+k)$-tic $F_{\eta_{1}, \ldots, \eta_{k}} \in \mathbb{C}\left[x_{0}, \ldots, x_{N-k}\right]$.

First of all, up to changing coordinates, we can assume that $\eta_{1}=\partial_{N-k+1}, \ldots, \eta_{k}=$ $\partial_{N}$. Then $\frac{\mathbb{C}\left[\partial_{0}, \ldots, \partial_{N}\right]}{\left(I(X), \partial_{N-k+1}, \ldots, \partial_{N}\right)}$, is Artinian Gorenstein, and can be considered as a 
quotient of the polynomial ring $\mathbb{C}\left[\partial_{0}, \ldots, \partial_{N-k}\right]$. Up to a change of coordinates, we can suppose that $F_{\partial_{N-k+1}, \ldots, \partial_{N}}:=x_{0}^{s+k}+\cdots+x_{N-k}^{s+k}$, and we can think of it as a polynomial in $\mathbb{C}\left[x_{0}, \ldots, x_{N-k}\right]$. We now find $F_{\partial_{N-k+1}, \ldots, \partial_{N}}^{\perp}$ as an ideal in $\mathbb{C}\left[\partial_{0}, \ldots, \partial_{N-k}\right]$. It is easy to see that

$$
F_{\partial_{N-k+1}, \ldots, \partial_{N}}^{\perp}=\left(\partial_{i} \partial_{j}, \partial_{i}^{s+k}-\partial_{j}^{s+k}\right), \quad i, j \in\{0, \ldots, N-k\}, \quad i \neq j,
$$

since, by hypothesis, $s+k>2$. Then the quadrics of $I(X)$ are of the form

$$
Q_{i, j}:=\partial_{i} \partial_{j}+\partial_{N-k+1} L_{i, j}^{1}+\cdots+\partial_{N} L_{i, j}^{k},
$$

where the $L_{i, j}^{\ell}$ 's are linear forms on $\check{\mathbb{P}}^{N}$. In particular, the vector space of the quadrics vanishing on $X$ has dimension $\left(\begin{array}{c}N-k+1 \\ 2\end{array}\right)$; that is: $h^{0}\left(\mathbb{P}^{N}, \mathcal{I}_{X}(2)\right)=\left(\begin{array}{c}N-k+1 \\ 2\end{array}\right)$. Since $X$ is PN it follows $(k+1) N-\left(\begin{array}{c}k \\ 2\end{array}\right)+1=h^{0}\left(X, \mathcal{O}_{X}(2)\right)$. Let $C$ be a general curve section of $X$. Hence $3 N-3 k+6=h^{0}\left(C, \mathcal{O}_{C}(2)\right)$ and we can proceed as in Castelnuovo's analysis of curves of maximal genus often called Castelnuovo curves: see for example GH94, pages 527-533]. In fact, since $C$ is non-degenerate, no quadric containing $C$ can contain a hyperplane $H \cong \check{\mathbb{P}}^{N+1-k}$, and therefore, if we set $\gamma:=C \cap H$, the natural restriction map $H^{0}\left(\mathbb{P}^{N+2-k}, \mathcal{I}_{C}(2)\right) \rightarrow H^{0}\left(H, \mathcal{I}_{\gamma}(2)\right)$ is an isomorphism (recall that $C$ is also linearly normal).

From this, we infer that the points of $\gamma$ impose only $h^{0}\left(H, \mathcal{O}_{H}(2)\right)-h^{0}\left(H, \mathcal{I}_{\gamma}(2)\right)=$ $2(N+2-k)-1$ conditions on quadrics. By Clifford's Theorem, $h^{0}\left(C, \mathcal{O}_{C}(2)\right)-1 \leq$ $\operatorname{deg}(C)$, therefore $\operatorname{deg}(C) \geq 3(N+2-k)-1>2(N+2-k)+1$ since $N>\bar{k}$. By Castelnuovo's Lemma (see for example [ACGH85, page 120]), if $H$ is generic, it follows that $\gamma$ is contained in a unique rational normal curve $D$.

Since $\gamma$ consists of more than $2 \operatorname{deg}(D)=2(N+2-k)-2$ points, a quadric contains $\gamma$ if and only if it contains $D$. Finally, we recall that a rational normal curve in $\mathbb{P}^{N+1-k}$ is the intersection of $\left(\begin{array}{c}N+1-k \\ 2\end{array}\right)$ quadrics, and therefore the intersection of the quadrics containing $X$ meets $H \cong \mathbb{P}^{N+1-k}$ exactly in $D$. Thus, the intersection of the quadrics containing $X$ is an irreducible $k$-dimensional variety $Y$ of minimal degree. By Bertini's classification theorem of the varieties of minimal degree, see EI85, it follows that $Y$ is a quadric or a RNS or a $k$-dimensional cone on the Veronese surface $v_{2}\left(\mathbb{P}^{2}\right)$.

Remark 4. The hypothesis of Theorem 23 concerning $s$ cannot be weakened; in fact, consider the del Pezzo surface $(Y, \mathcal{L})=\left(\mathbb{P}^{2}, \mathcal{O}_{\mathbb{P}}(3)\right)$. Let $X:=j(Y)$ where $j:=\phi_{|H|} . \quad X \subset \mathbb{P}^{9}$ is $(-1)-a G$ but since $X$ does not contain plane curves, it cannot be contained in a rational normal threefold of $\mathbb{P}^{9}$.

Remark 5. As we noted in the introduction, the hypothesis $k>2$ is crucial, in the sense that if $k=2$ there are subcanonical curves contained in rational normal scrolls which are not PN. For example, take a smooth curve $C \in\left|5\left(C_{0}+f\right)\right|$ on the rational normal scroll $S_{1,2} \subset \mathbb{P}^{4}$, where $C_{0}^{2}=-1$ is the section at infinity and $f$ is the fibre. Then $C$ is 1 -subcanonical of genus 6 and degree 10: it is a projection of a canonical curve of $\mathbb{P}^{5}$, and therefore $C$ is not linearly normal.

Corollary 24. If $X$ is a $(k-1)$-dimensional s-subcanonical variety with crepant resolution contained as a divisor in a variety of minimal degree $S$ such that $s+k>2$, such that $X$ is not aCM, then $k=2$, i.e. $X$ is a curve and $S$ is a rational normal scroll $S_{a_{1}, a_{2}}$ with $a_{1} \neq a_{2}$.

Proof. This follows from Lemma 16, Corollary 14, Proposition 22 and Theorem 23. 
3.6. The case $s+k=2$. We recall that a del Pezzo variety is a pair $(X, H)$, where $X$ is a projective $n$-dimensional variety $X$ with only Gorenstein (not necessarily normal) singularities and $H$ is an ample Cartier divisor on it such that $-K_{X}=$ $(n-1) H$, and $h^{i}\left(X, \mathcal{O}_{X}(j H)\right)=0$, for all $i, j \in \mathbb{Z}$ with $0 \leq i \leq n$.

For example, by the Kawamata-Viehweg theorem, if $X$ is a Gorenstein Fano variety of index $n-1$ with at most canonical singularities, it is a del Pezzo variety.

For a normal Gorenstein del Pezzo variety $X$ of codimension $e \geq 2$ it clearly holds that $s+k=2$, but our Theorem 23 fails in this case for trivial reasons since in Equation 11 the terms $\partial_{i}^{s+k}-\partial_{j}^{s+k} i \neq j$ and $i, j=0, \ldots, N-k$ are quadratic. Following the argument of the proof we can deduce that the homogeneous ideal of these projective varieties is generated by quadrics.

Finally we remark that if $X$ is a del Pezzo variety contained in a RNS, using the notation of Subsection [3.5, it easily follows that either $X \in|2 H+(2-f) F|$ or $X$ is a hypercubic in the trivial case $k=N, S_{a_{1}, \ldots, a_{k}}=\mathbb{P}^{N}$. In the first case, $j^{-1}(X) \in\left|3 H-H_{0}\right|$ and this is a case of Fujita's classification see Fuj90, Theorem 9.17 page 82 ] of the del Pezzo varieties.

\section{REFERENCES}

[ACGH85] E. Arbarello, M. Cornalba, P. Griffiths, and J. Harris, Geometry of algebraic curves. Vol. I, Grundlehren der Mathematischen Wissenschaften [Fundamental Principles of Mathematical Sciences], vol. 267, Springer-Verlag, New York, 1985.

[AS78] E. Arbarello and E. Sernesi, Petri's approach to the study of the ideal associated to a special divisor, Invent. Math. 49 (1978), no. 2, 99-119.

[BCN11] E. Ballico, G. Casnati, and R. Notari, Canonical curves with low apolarity, J. Algebra 332 (2011), 229-243.

[BH93] W. Bruns and J. Herzog, Cohen-Macaulay rings, Cambridge Studies in Advanced Mathematics, 39. Cambridge University Press, Cambridge, 1993.

[CRV01] A. Conca, M. E. Rossi and G. Valla, Gröbner flags and Gorenstein algebras Compositio Math. 129 (2001), no. 1, 95-121.

[DZ11] P. De Poi and F. Zucconi, Gonality, apolarity and hypercubics, Bull. London Math. Soc. 43 (2011), 849-858.

[DZ2] Fermat hypersurfaces and Subcanonical curves, Internat. J. Math. 22 (2011) no. $12,1763-1785$.

[EI85] D. Eisenbud and J. Harris, On varieties of minimal degree. (A centennial account), Algebraic geometry, Proc. Summer Res. Inst., Brunswick/Maine 1985, part 1, Proc. Symp. Pure Math. 46, 3-13 (1987).

[Fer01] R. Ferraro, Weil divisors on rational normal scrolls, Geometric and combinatorial aspects of commutative algebra (Messina, 1999), 183-197, Lecture Notes in Pure and Appl. Math., 217, Dekker, New York, 2001.

[Fuj90] T. Fujita, Classification theories of polarized varieties, London Mathematical Society Lecture Note Series, 155. Cambridge University Press, Cambridge, 1990.

[Ger96] A. V. Geramita, Inverse System of fat points: Waring Problem, Secant Varieties and Veronese Varieties and Parametric Spaces of Gorenstein Ideals, Queen's Papers in Pure ans applied Mathematics no. 102, The Curves seminar at Queen's (1996), Vol. X, 3-114.

[GR70] H. Grauert and O. Riemenschneider, Verschwindungssätze für analytische Kohomologiegruppen auf komplexen Räume, Invent. Math. 11 (1970), 263-292.

[Gre82] M. L. Green, The canonical ring of a variety of general type, Duke Math. J. 49 (1982) no. 4, 1087-1113.

[GH94] P. Griffiths and J. Harris Principles of algebraic geometry. Wiley Classics Library. John Wiley \& Sons, Inc., New York, 1994, Reprint of the 1978 original.

[Har83] R. Hartshorne, Algebraic geometry, Graduate Texts in Mathematics, vol. 52, SpringerVerlag, New York-Heidelberg-Berlin, 1983, Corr. 3rd printing.

[IK99] A. Iarrobino and V. Kanev, Power sums, Gorenstein algebras, and determinant loci, Lect. Notes Math., vol. 1721, Springer, Berlin, 1999.

[Kol95] J. Kollár, Singularities of pairs, Algebraic geometry-Santa Cruz 1995, 221-287, Proc. Sympos. Pure Math., 62, Part 1, Amer. Math. Soc., Providence, RI, 1997. 
[Mac16] F. S. Macaulay, The algebraic theory of modular systems. With a new introduction by Paul Roberts, Reprint of the 1916 orig. Cambridge Mathematical Library. Cambridge: Cambridge University Press, (1994).

[Mig98] J. C. Migliore, Introduction to liaison theory and deficiency modules, Progress in Mathematics, vol. 165, Birkhäuser, Boston, Mass., 1998.

[Rei83] M. Reid, Minimal models of canonical threefolds, Algebraic Varieties and Analytic Varieties, Tokyo, 1981, Adv. Stud. Pure Math. Vol. 1, ed. S. Iitaka, (1983) 131-180.

[Sch86] F. Schreyer, Syzygies of canonical curves and special linear series, Math. Ann. 275 (1986), 105-137.

[Vak06] R. Vakil, Murphy's law in algebraic geometry: badly-behaved deformation spaces, Invent. Math. 164 (2006), no. 3, 569-590.

Dipartimento di Matematica e Informatica, Università degli Studî di Udine, Via delle Scienze, 206, Loc. Rizzi, 33100 Udine, Italy

E-mail address: pietro.depoi@uniud.it

E-mail address: francesco.zucconi@uniud.it 